\title{
Lightning striking twice: Redo mitral valve replacement due to recurrent cardiac sarcoma using partial autotransplantation technique
}

\author{
Basil Al-Sabeq, MD, ${ }^{a}$ Steven S. Shen, MD, PhD, ${ }^{\mathrm{b}}$ Michael Reardon, MD, ${ }^{\mathrm{c}}$ and Faisal Nabi, MD, ${ }^{\mathrm{a}}$ \\ Houston, Tex
}

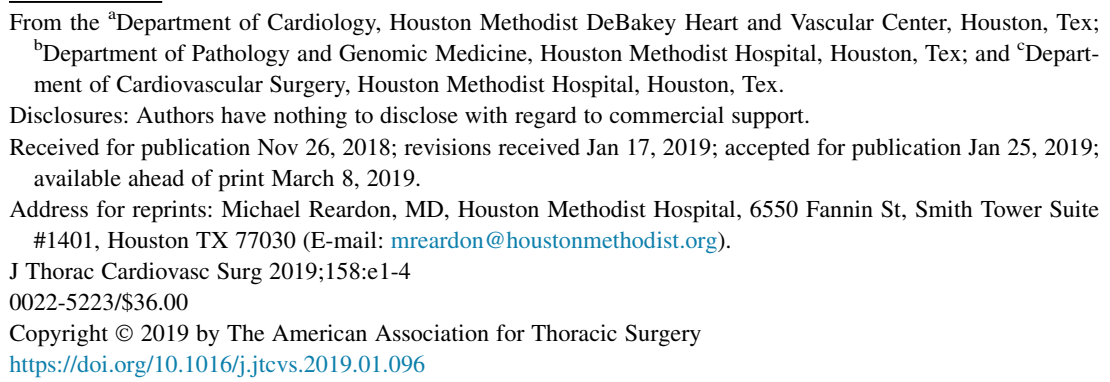

$\checkmark$ Video clip is available online.

Although exceptionally rare, sarcomas are the most common primary malignant cardiac tumors. ${ }^{1}$ Angiosarcomas are the most common among various histologic subtypes, ${ }^{2}$ but all subtypes are almost invariably associated with poor outcomes due to their aggressive nature. We report a case of an undifferentiated cardiac sarcoma recurrence on a bioprosthetic mitral valve leading to severe inflow obstruction, pulmonary hypertension, and heart failure.

\section{CASE DESCRIPTION}

A 37-year-old woman was hospitalized for progressive functional decline and exertional dyspnea resulting in heart failure. Her surgical history was notable for left atrial sarcoma resection with microscopically positive margins and concomitant bioprosthetic mitral valve replacement due to tumor extension 10 months previously. At that time, computed tomography had revealed sclerotic lesions within the axial skeleton concerning for metastases. The patient declined proposed chemotherapy with doxorubicin, ifosfamide, and the cardioprotective agent dexrazoxane.

The patient presented to her treating physician with severe shortness of breath at minimal activity. She did not report fevers or constitutional symptoms, was afebrile, and had negative blood cultures. Transthoracic echocardiography revealed preserved left ventricular systolic 70-75 mm Hg.

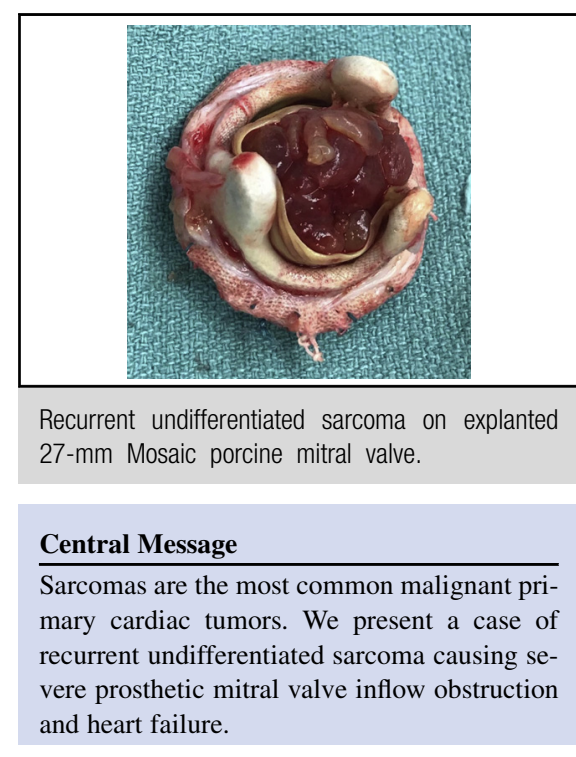

See Commentary on page e5.

function and a large, $2.4 \times 2.2-\mathrm{cm}$ echogenic mass attached to the mitral prosthesis and prolapsing into the left ventricle, causing severe mitral inflow obstruction (Figure 1, $C$, and Video 1). The calculated diastolic mean transmitral gradient using continuous-wave Doppler was $24 \mathrm{~mm} \mathrm{Hg}$ at a heart rate of 85 beats per minute (Figure 1,D). Estimated right ventricular systolic pressure was significantly elevated at

Due to suspicion for cardiac sarcoma recurrence, a cardiac magnetic resonance study was performed. This revealed a large mass on the mitral bioprosthesis (Figure 1, $A$ and $B$, and Video 2), but attempts at tissue characterization were compromised due to the rapid motion of the lesion and the presence of the bioprosthesis. In addition, the right ventricle was severely dilated (right ventricular end-diastolic volume index $165 \mathrm{~mL} / \mathrm{m}^{2}$ ) with severe global systolic dysfunction (right ventricular ejection fraction $24 \%$ ) in the setting of a dilated main pulmonary artery $(3.6 \mathrm{~cm})$.

The patient was referred to cardiothoracic surgery at our institution for resection of a suspected recurrent sarcoma due to her New York Heart Association functional class 


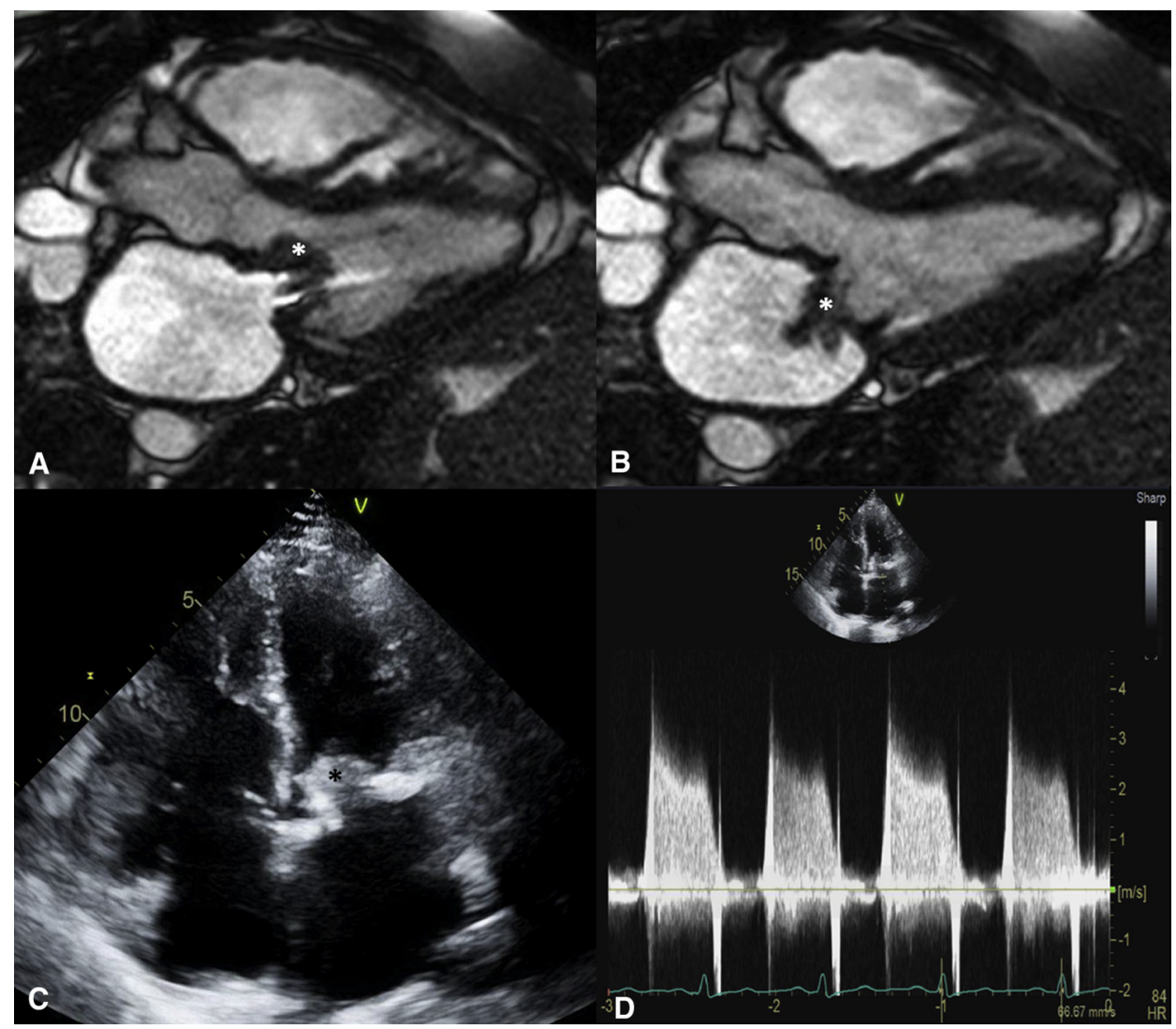

FIGURE 1. Cardiac magnetic resonance balanced steady-state free precession 3-chamber long axis cine images showing the bioprosthetic mitral valve sarcoma prolapsing in diastole (A) and systole (B). Apical 4-chamber transthoracic echocardiogram view shows the sarcoma during diastole (C). Transmitral continuous wave Doppler (D) showing severely elevated transmitral gradients (peak/mean gradients, $55 / 24 \mathrm{~mm} \mathrm{Hg}$ ). Asterisks indicate the tumor.

IV heart failure. Her case was reviewed by the multidisciplinary cardiac tumor team (MD Anderson Cancer Center and Houston Methodist Hospital), and palliative resection

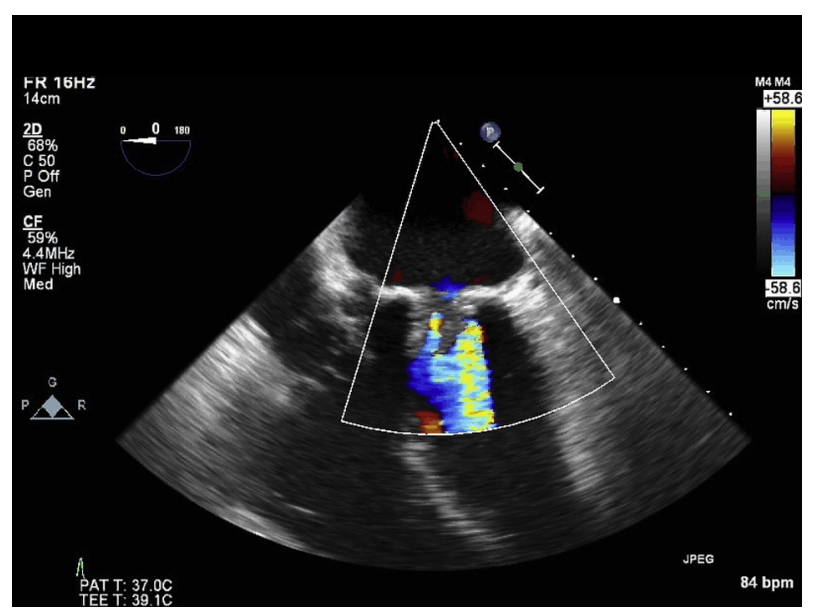

VIDEO 1. Perioperative transesophageal echocardiogram 4-chamber view at the midesophageal level reveals the prolapsing sarcoma and significant transmitral flow acceleration with color Doppler. Again, a dilated and significantly dysfunctional right ventricle is noted. Video available at: https://www.jtcvs.org/article/S0022-5223(19)30311-3/fulltext. and adjuvant chemotherapy with doxorubicin (Adriamycin; Pfizer Inc, New York, NY) and ifosfamide were recommended. She underwent a redo sternotomy, removal of a 27-mm Mosaic porcine mitral valve (Medtronic, Minneapolis, Minn), redo mitral valve replacement with a 29$\mathrm{mm}$ Mosaic bioprosthesis, and partial resection of the right inferior pulmonary vein and left atrial tissue due to suspected tumor invasion. A partial autotransplantation technique with division of the pulmonary artery, aorta, and superior vena cava but leaving the inferior vena cava intact was used to gain wider exposure to the left atrium and minimize the risk of tumor embolization. Histopathology of the explanted valve and pulmonary vein revealed findings consistent with a high-grade, undifferentiated sarcoma (Figure 2). Postoperative echocardiography revealed a normal gradient across the mitral valve of $6 \mathrm{~mm} \mathrm{Hg}$ at a heart rate of 91 beats per minute.

The patient's postoperative course was uneventful, and she was discharged on postoperative day 7 . She returned to her home out of state in functional class I but refused chemotherapy. Follow-up by e-mail 4 months after surgery confirmed recurrence of the tumor in the left ventricle by echocardiogram. The patient died 5 and a half months after surgery without return of heart failure until the last month. 


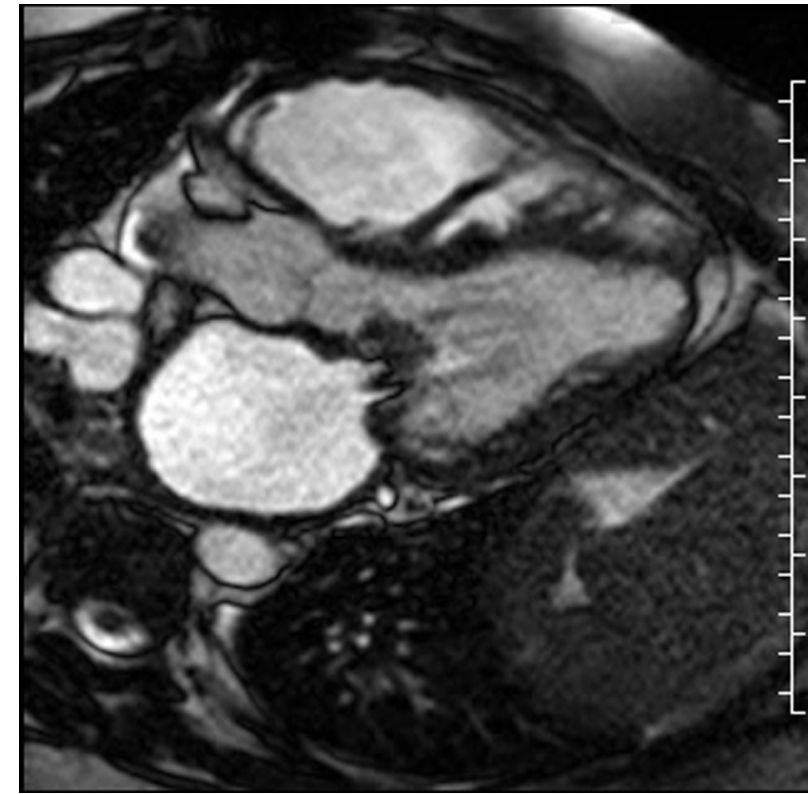

VIDEO 2. High-resolution balanced steady-state free precession 3-chamber long axis cine showing sarcoma on the mitral bioprosthesis prolapsing in and out of the left ventricle. Flow acceleration and turbulence across the mitral valve suggest inflow obstruction. A dilated, dysfunctional right ventricle is also appreciated. Video available at: https://www.jtcvs.org/article/S00225223(19)30311-3/fulltext.

\section{DISCUSSION}

Although cardiac sarcomas are the most common primary malignant cardiac tumors, they remain extremely rare. They are almost uniformly and rapidly lethal without surgical management, ${ }^{2}$ with the large surgical case series of 95 patients from our tumor group demonstrating a postoperative mortality rate of $35 \%$ at 1 year. ${ }^{1}$ Undifferentiated pleomorphic sarcomas are a particularly rare histologic subtype that tend to have a predilection for the left atrium and invasion into mitral valve tissue. ${ }^{2-4}$ Similar to our case, severe mitral inflow obstruction leading to heart failure, pulmonary hypertension, and valve replacement has been reported, as has recurrence on mitral valve prostheses. Their tendency to occur in the left atrium attached to the interatrial septum may invoke the more common and "benign" cardiac myxoma, ${ }^{2}$ and tissue characterization with noninvasive imaging to differentiate the 2 remains challenging and unsatisfactory for a definitive diagnosis.

In our case, surgical tumor resection was indicated due to clinical heart failure, and the index of suspicion for malignant tumor recurrence was already raised due to the patient's history. Cardiac autotransplantation technique can be used to gain the needed exposure to resect and reconstruct posterior heart tumors such as sarcomas. ${ }^{5}$ In this case of palliative resection, we divided the superior vena cava and both great vessels, leaving the inferior vena cava intact. This partial autotransplantation technique allowed wide exposure of the left atrium for extensive tumor resection and reconstruction while maintaining the orientation of the heart to simplify the procedure. Our case highlights the typical behavior of this rare cardiac pathology, serves as a reminder to think broadly in the evaluation of cardiac masses, demonstrates the potential value of multimodality imaging in the evaluation of cardiac masses, and highlights an approach for simplified extensive left atrial resection.

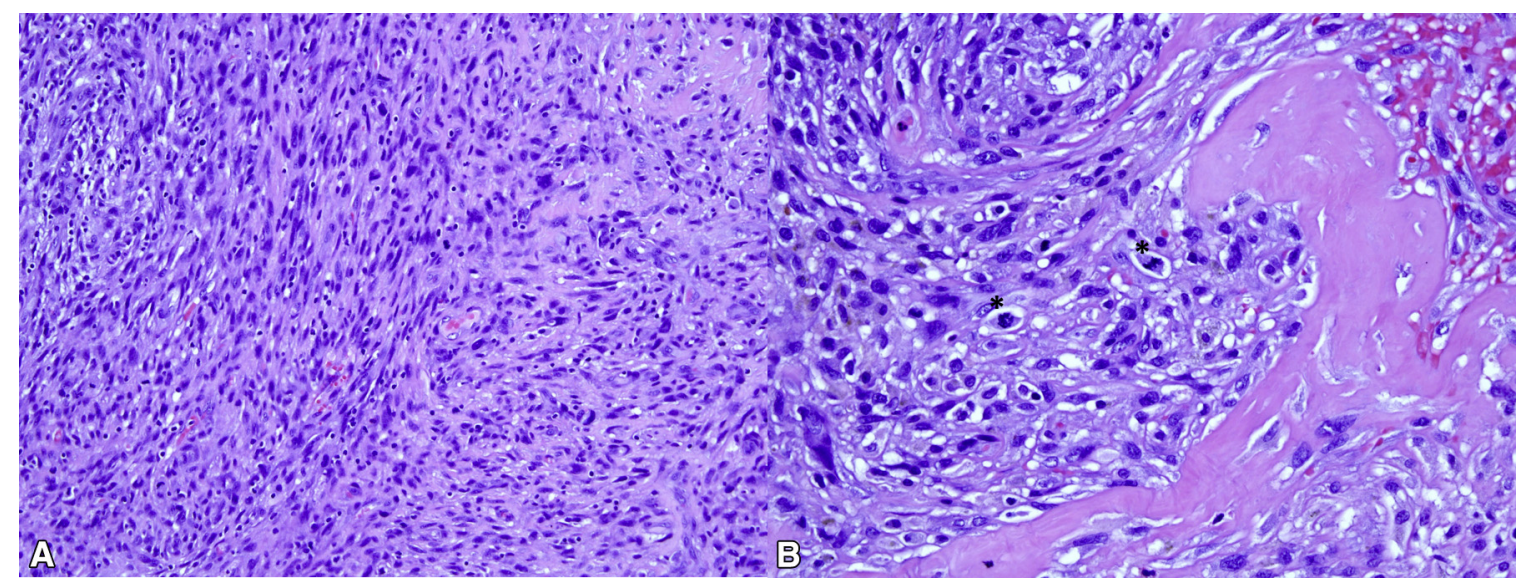

FIGURE 2. Histopathology of mitral valve tissue at $10 \times$ magnification showing focal hypercellular spindle and epithelioid cells characteristic of undifferentiated sarcoma (A). Large, pleomorphic nuclei and prominent nucleoli and mitotic activity (asterisks) at $20 \times$ magnification (B) signify rapidly dividing tumor cells. 
This case also highlights the need for biologic systemic treatment in addition to surgery.

\section{References}

1. Ramlawi B, Leja MJ, Abu Saleh WK, Al Jabbari O, Benjamin R, Ravi V, et al. Surgical treatment of primary cardiac sarcomas: review of a single-institution experience. Ann Thorac Surg. 2016;101:698-702.

2. Valles-Torrés J, Izguierdo-Villarroya MB, Vallejo-Gil JM, CasadoDomínguez JM, Roche Latasa AB, Auquilla-Clavijo P. Cardiac undifferentiated pleomorphic sarcoma mimicking left atrial myxoma. J Cardiothorac Vasc Anesth. 2019:33:493-6.

3. Watson R, Frye J, Trieu M, Yang MX. Primary undifferentiated pleomorphic cardiac sarcoma with MDM2 amplification presenting as acute left-sided heart failure. $B M J$ Case Rep. https://doi.org/10.1136/bcr-2018-226073. Accessed September 30, 2018.

4. Aguilar C, Soca R, Guillen M, Paucca E, Illatopa V. Cardiac undifferentiated pleomorphic sarcoma incidentally diagnosed during mitral valve replacement. J Card Surg. 2017;32:91-2.

5. Ramlawi B, Al-Jabbari O, Blau LN, Davies MG, Bruckner BA, Blackmon SH, et al. Autotransplantation for the resection of complex left heart tumors. Ann Thorac Surg. 2014;98:863-8. 